\title{
Arterial desaturation dependent on posture
}

\author{
L. D. A B R A M S, R. B. COLE, G. CUMMING, L. K. HARDING, \\ E. HOUSLEY, ANDK. PROWSE \\ From the Department of Medicine, Queen Elizabeth Hospital, Birmingham
}

\begin{abstract}
A 60-year-old woman developed subacute bacterial endocarditis following a minor operation. She presented the unusual picture of breathlessness with cyanosis related to posture, confirmed by laboratory measurement. She had an atrial septal defect with right-to-left shunt, and had sustained multiple pulmonary infarcts, resulting in the obliteration of a large section of her pulmonary vascular bed. The puzzling absence of pulmonary hypertension was explained by tricuspid incompetence, although there was no clinical evidence of this, and on two occasions the right atrial pressure tracing was normal. The use of a balloon catheter to predict the results of operation is described. At operation the severely damaged tricuspid valve was replaced, and the septal defect was closed. There was an immediate improvement in the arterial oxygen tension after operation. She subsequently survived an episode of ventricular fibrillation, left hospital 11 months after her original admission and is now leading a normal life.
\end{abstract}

The occurrence of central cyanosis in a patient with an intracardiac shunt is normally due to an increase in right heart pressures, and is clearly understood.

The transient development of this sign associated with an alteration in posture is less common, and the case we are describing involves a combination of circumstances producing this clinical sign.

\section{CASE HISTORY}

In December 1966, two weeks after an operation for removal of a fibro-adenoma of the breast, a 60-yearold woman developed general malaise with rigors and vomiting. In February 1967 she was noted to be febrile and to have an apical systolic murmur. There was no history of rheumatic fever. Blood and urine cultures grew Proteus vulgaris but treatment with a number of antibiotics, alone and in combination, produced only a slight improvement. During relapses her temperature rose to $104^{\circ} \mathrm{F}$. $\left(40^{\circ} \mathrm{C}\right.$.). In July she complained of breathlessness when sitting up and subsequently this was observed to be accompanied by cyanosis. The breathlessness and cyanosis disappeared on lying down. In July and August she had two episodes of pleuritic chest pain. During this period she became breathless and cyanosed when lying down, these signs being more marked when she sat up.

These clinical observations were confirmed by the following values of arterial oxygen saturation which also showed that desaturation was unaffected by breathing oxygen. The results were as follows:
Supine, breathing air

Supine, after breathing $100 \%$ oxygen for $5 \mathrm{~min}$.

Sitting, breathing air

Arterial Oxygen Saturation (\%) 68 68 59

These results indicate the presence of a central shunt which increased on sitting up. During the period May to August, electrocardiography sometimes showed sinus rhythm and at other times atrial fibrillation.

On 23 August 1967 she was transferred to the Queen Elizabeth Hospital, Birmingham, for further investigation. On admission she was ill and emaciated. Breathlessness and cyanosis were marked when she was lying flat but both signs increased when she sat up, and were associated with dizziness and faintness after sitting for 15 seconds. Finger clubbing was present. The pulse rate was $128 /$ minute, there were no signs of congestive cardiac failure, and there was no left parasternal heave. The heart sounds were normal but a soft precordial systolic murmur was present, maximal in the second intercostal space at the left sternal border.

The haemoglobin was $14 \cdot 3 \mathrm{~g} . / 100 \mathrm{ml}$.; white cell count $12,200 /$ cu.mm. ; and E.S.R. $26 \mathrm{~mm}$. in the first hour (Westergren). An electrocardiogram showed sinus rhythm with no evidence of right ventricular hypertrophy. A chest radiograph showed prominenoe of the pulmonary vascular pattern in the right lung, particularly in the lateral basal segment and in the upper lobe. The outline of the heart was normal. Blood cultures were negative, including those taken directly from the right side of the heart during catheterization, 
but nevertheless a diagnosis of subacute bacterial endocarditis was made on clinical grounds. Her general condition deteriorated rapidly and she was given a continuous intravenous infusion of penicillin in a dose of $\mathbf{4 0}$ megaunits daily for six weeks. After two weeks of treatment the rigors stopped and by the end of the third week the fever, which had been present for eight months, abated.

At this stage further investigations were made into the right-to-left shunt which appeared to be responsible for the patient's cyanosis. Right heart catheterization was carried out on 1 September 1967. Serial oxygen saturations showed no evidence of a left-toright shunt but dye curves indicated a right-to-left shunt at atrial level, calculated to be $40 \%$ of the pulmonary blood flow. This agreed closely with that calculated from the arterial oxygen saturation (35\%), making a significant intrapulmonary shunt unlikely.

The pressures recorded were within normal limits (Table I). The pressure values given in the Table are in conventional units in the first column and in Standard International units in the second column.

\section{T A B L E I}

\begin{tabular}{|c|c|c|c|c|}
\hline & & & $\begin{array}{r}\text { Pressure } \\
(\mathrm{mm} . \mathrm{Hg})\end{array}$ & $\mathrm{kN} \mathrm{m}^{-2}$ \\
\hline $\begin{array}{l}\text { Pulmonary artery } \\
\text { Right ventricle .. }\end{array} \quad \ldots$ & $\begin{array}{l}. \\
\because \\
\because\end{array}$ & $\begin{array}{l}. \\
\because \\
\because\end{array}$ & $\begin{array}{l}19 / 8 \\
22 / 1 \\
6 \\
7\end{array}$ & $\begin{array}{c}2 \cdot 54 / 1 \cdot 07 \\
2 \cdot 94 / 0 \cdot 13 \\
0 \cdot 80 \\
0.93\end{array}$ \\
\hline
\end{tabular}

She was too ill to perform exercise. The wave form in the right atrium was normal. The cardiac output was $5.61 . / \mathrm{min}$. (dye curve) and the brachial artery oxygen saturation was $72 \%$ breathing air and $81 \%$ breathing pure oxygen, suggesting that the shunt remained the dominant cause of desaturation.

During September she had episodes of pleuritic pain, both on the left and on the right side, associated on one occasion with haemoptysis, and intravenous heparin was added to the penicillin. In view of the repeated episodes of pulmonary infarction a lung scan (Picker Magnascanner) was undertaken using macro-agglutinated ${ }^{131}$ I labelled human serum albumin. The radioactivity in horizontal strips of the colourscan over the whole chest was assessed by counting the number of dots of each colour per strip and expressing it as a percentage of the total count. Figure 1 shows that the perfusion is poor in the lower zones, while Fig. 2 shows this to be more marked in the right lung compared with the left.

At this stage in the investigation the diagnosis appeared to be (a) subacute bacterial endocarditis ; (b) atrial septal defect with right-to-left shunt ; (c) pulmonary embolic disease.

The absence of pulmonary hypertension was remarkable and suggested the presence of tricuspid incompetence. Free flow of blood could thus occur through the incompetent valve and the septal defect during systole, with the maintenance of a normal diastolic pressure. However, there were no prominent ' $v$ ' waves in the jugular pulse, the liver was not enlarged, there was no evidence of right ventricular hypertrophy and no pansystolic murmur. Furthermore this diagnosis was not supported by the right atrial pressure tracings obtained at right heart catheterization. Since conventional methods had failed to demonstrate tricuspid incompetence, right heart angiography was carried out. Tricuspid incompetence was indeed present and the existence of a right-to-left interatrial shunt was confirmed. The right ventricular pressure was again normal $\left(20 / 2 \mathrm{~mm}\right.$. $\left.\mathrm{Hg} ; 2 \cdot 70 / 0.27 \mathrm{kN} \mathrm{m}^{-2}\right)$.

The final diagnosis, therefore, was (a) subacute bacterial endocarditis; (b) atrial septal defect with right-to-left shunt with mean atrial pressures within normal limits; (c) multiple pulmonary infarcts, with diminished perfusion in both lower zones; $(d)$ tricuspid incompetence.

In spite of the high risk, surgical treatment seemed to offer the only hope of a successful outcome. In an attempt to predict the effects of operation, right heart catheterization was again carried out on 9 November 1967, measurements being taken both before and after occlusion of the defect by a balloon. The balloon catheter was passed from the leg and went through a
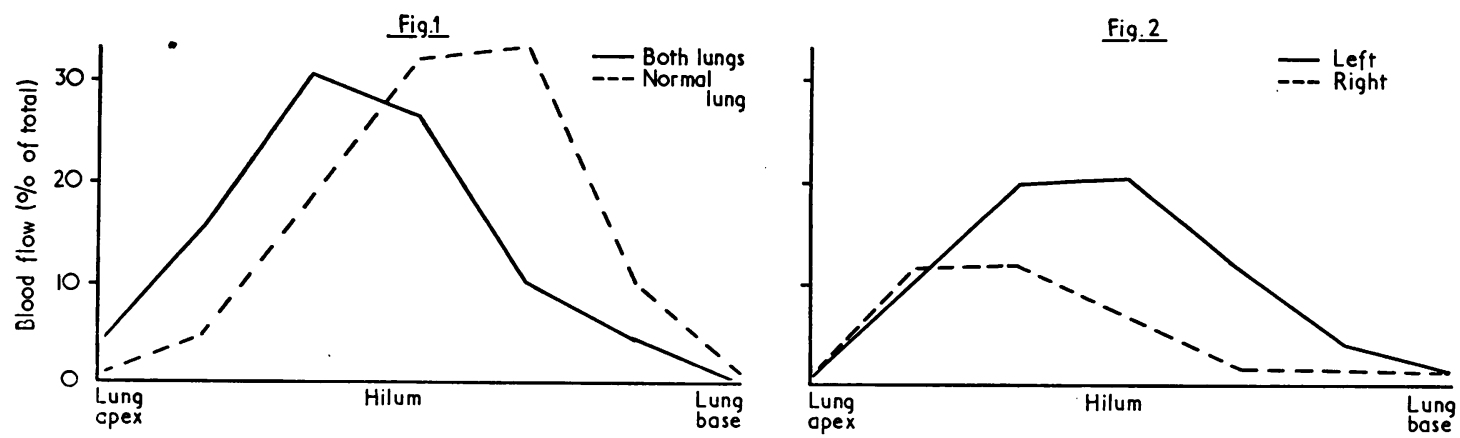

FIG. 1. Percentage distribution of pulmonary blood flow for both lungs taken together, compared with a normal similarly measured. FIG. 2. Percentage distribution of pulmonary blood flow in each lung taken separately. 
small high interatrial communication (probably a patent foramen ovale). Measurements were taken with the patient lying with the shunt open, lying with the shunt closed, sitting with the shunt closed, and finally sitting with the shunt open. When the shunt was closed by inflating the balloon her cyanosis and breathlessness improved and she felt better. On deflating the balloon her symptoms returned at once. The results are shown in Table II.

Exploration of the heart on cardiopulmonary bypass was undertaken on 17 November. Large quantities of organized thrombus were removed from the pulmonary artery to the right lower lobe. A foramen ovale defect, measuring $2 \times 1 \mathrm{~cm}$., was closed. The tricuspid valve was severely damaged, two-thirds of the anterior cusp were missing, the chordae tendineae were grossly shortened, and the valve showed vegetations and calcification. The valve was excised and replaced by a prosthesis.

In the immediate postoperative period she was not cyanosed, her arterial $\mathrm{Po}_{2}$ being $104 \mathrm{~mm}$. $\mathrm{Hg}(97 \cdot 5 \%$
T A B L E I I

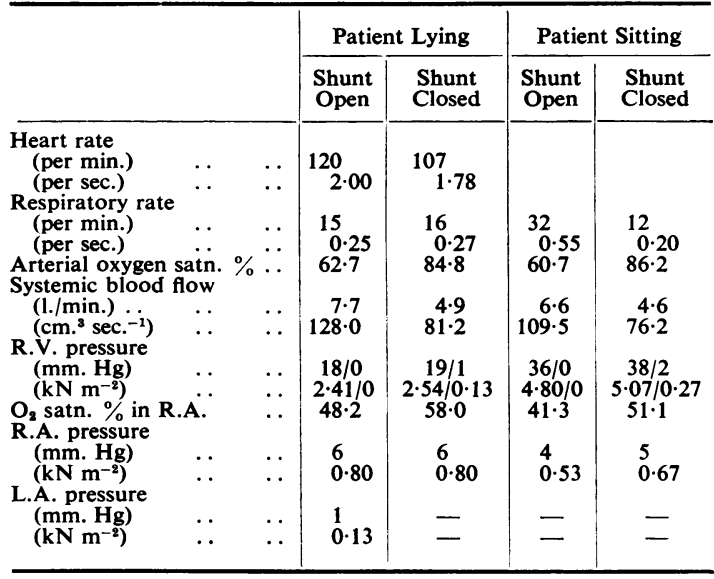

N.B. Values are given in conventional units and in Standard International units.

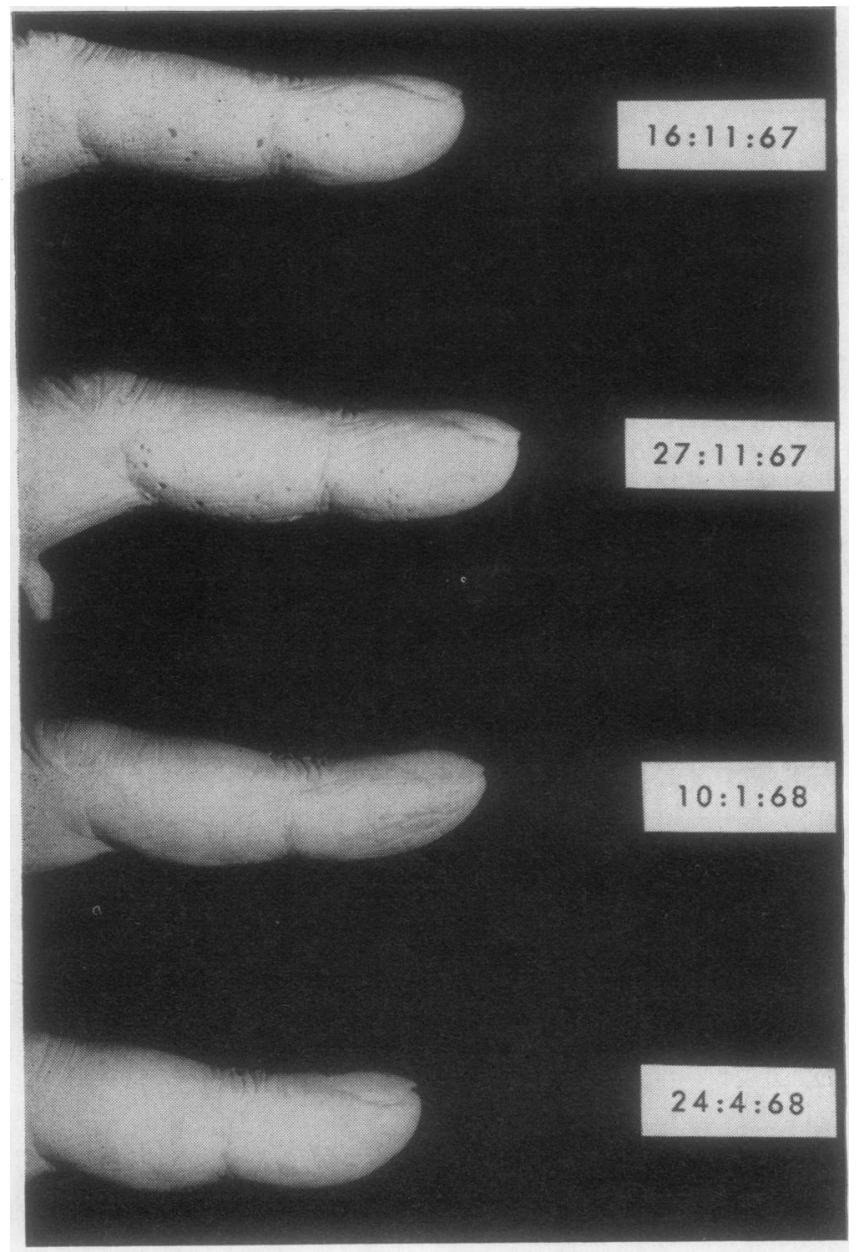

FIG. 3. The casts of an index finger taken at various times during and after the illness illustrate the regression of finger clubbing. 
saturation) breathing 4 litres of oxygen per minute through a nasal catheter (estimated inspired oxygen $35 \%)$. Cloxacillin and streptomycin were added to the intravenous regime of penicillin and heparin. Eighteen hours after operation she showed evidence of progressive peripheral circulatory failure culminating in a short period of ventricular fibrillation successfully treated by D.C. shock. Subsequently she required meohanical ventilation, with an intravenous adrenaline drip to maintain her blood pressure.

Her subsequent course was stormy but her improvement allowed return to the general ward four days after operation. Six days later she developed a pyrexia of $100^{\circ} \mathrm{F}$. $\left(38^{\circ}\right.$ C.) which lasted intermittently for eight days when the pyrexia subsided and all antibiotics, except penicillin V, $250 \mathrm{mg}$. four times a day, were stopped; the intravenous heparin therapy was replaced by oral phenindione.

In spite of the steady improvement in her physical condition she became severely depressed, requiring treatment with amitriptyline for six weeks. Mobilization was slow due to severe wasting and weakness of the muscles of the limbs and trunk resulting from her confinement to bed for almost one year, but exercises under the direction of the physiotherapist effected a slow but continuous improvement.

A further lung scan was carried out six weeks after operation to assess the state of the lower zone perfusion following the surgical disobliteration of the right lower lobe pulmonary artery but there was no change in the pattern of pulmonary blood flow. A comparison of finger casts taken pre- and postoperatively showed diminution in the extent of the finger clubbing. A further cast taken three months after operation was within normal limits (Fig. 3).

At the time of discharge, in January 1968, her limiting symptom was muscular weakness but she was able to walk 50 yards $(45 \mathrm{~m}$.) unaided. When seen in the outpatient department six weeks after discharge she was able to do housework, was free from symptoms, and had regained her normal weight.

\section{DISCUSSION}

The finding of only three positive blood cultures in this patient with subacute bacterial endocarditis was not surprising as the endocarditis was predominantly right-sided. Several regimes of treatment which took into account the bacterial sensitivities were unsuccessful. Eventually the conventional treatment using high doses of penicillin by continuous intravenous infusion proved successful, indicating the efficacy of this relatively safe form of treatment and the importance of continuing treatment for a sufficient period of time.

The source of the repeated pulmonary emboli is a matter for conjecture, possible sites being vegetations in the right side of the heart and thrombosis in the deep veins of the legs and pelvis. The danger of anticoagulant therapy in the presence of emboli from bacterial vegetations is well recognized but in this case no ill effects were noted.

In the normal upright lung, the lower lobes are well perfused and the upper lobes poorly perfused, under the influence of gravity. In lungs with impairment of dependent flow due to obliteration of the pulmonary vascular bed, perfusion is maintained by an increase in pulmonary artery pressure, so that blood flows through the upper lobes even in the upright position.

The presence of an atrial septal defect is usually associated with a high pulmonary blood flow and pulmonary hypertension, but the maintenance of a high systolic ejection pressure in the right ventricle is compromised by an incompetent tricuspid valve.

Thus in this patient the assumption of the erect position, with its requirement for an increased right ventricular systolic pressure in order to maintain upper lobe perfusion, resulted in an increase of blood flow into the atrium, an increase in the right-to-left shunt, and failure to increase right heart pressures. The cyanosis therefore became more marked on sitting up, and was little changed by breathing pure oxygen.

Despite right heart catheterization on three occasions, with pressure recordings in the right atrium, and repeated clinical examination, the diagnosis of tricuspid incompetence was made only after right-sided angiography, and even this investigation failed to give an accurate picture of the extent of valve destruction found at operation.

This evidence suggests that the absence of the characteristic fusion of $a$ and $c$ waves in the right atrium pressure record does not negate the diagnosis of tricuspid incompetence. 